\title{
TI.107.1
}

\section{Baseline Expectations Community Consensus Process}

- PDF: TI.107.1-BaselineExpectationsCommunityConsensusProcess.pdf

- Text: TI.107.1-BaselineExpectationsCommunityConsensusProcess.txt

\section{More Information}

\begin{tabular}{|c|c|}
\hline Repository ID & TI.107.1 \\
\hline Persistent URL & http://doi.org/10.26869/TI.107.1 \\
\hline Title & Baseline Expectations Community Consensus Process \\
\hline Authors & InCommon CTAB \\
\hline Sponsor & InCommon Community Trust and Assurance Board (CTAB) \\
\hline Review & Community Consultation \\
\hline Status & Preserve \\
\hline Publish Date & July 2018 \\
\hline $\mathrm{DOI}$ & 10.26869/TI.107.1 \\
\hline \multicolumn{2}{|l|}{ Signature } \\
\hline Deprecated & No \\
\hline \multicolumn{2}{|l|}{ Future Review } \\
\hline Supersedes & NA \\
\hline Format & PDF, Text \\
\hline \multicolumn{2}{|l|}{ Related Docs } \\
\hline Development Location & https://docs.google.com/document/d/1Ao-ZO9hfWMuSh0KHMegZV3S8ETS1jM1hlk8hmy_UjFl/edit \\
\hline \multicolumn{2}{|l|}{ IP Framework } \\
\hline Subject Tags & federation, trust, assurance, ctab \\
\hline Notes & \\
\hline
\end{tabular}

